\title{
La teoría de la sociedad internacional. De la narrativa clásica de la Escuela Inglesa al enfoque crítico
}

\section{ELSA AIMÉ GoNZÁLEZ}

$\mathrm{E}$

n los últimos años se han vuelto a editar y a estudiar tres clásicos de la Escuela Inglesa de las Relaciones Internacionales. En 2012 salió la cuarta edición de The Anarchical Society, publicada por primera vez en 1977, con un nuevo prefacio escrito por Andrew Hurrell, mientras que en 2017 se celebraron los cuarenta años de dicha obra con un volumen editado por Hidemi Suganami, Madeleine Carr y Adam Humphreys en la que se han planteado varios debates para repensar este clásico escrito por Hedley Bull.' En España la obra Cambios en la Naturaleza de la Diplomacia y de la Guerra en los Cuarenta Años de la Sociedad Anárquica de Hedley Bull publicado en 2019 también ha conmemorado este aniversario. ${ }^{2}$ Asimismo, está prevista la publicación de una nueva edición de la obra Diplomatic Investigations, publicada por primera vez en 1966, con una nueva introducción de Tim Dunne e lan Hall, así como de la obra colectiva The Expansion of International Society publicada en 1984 con un nuevo prefacio de Andrew Hurrell. ${ }^{3}$

Estas tres obras son características (aunque no son las únicas) de lo que denominamos la Escuela Inglesa clásica o teoría clásica de la sociedad internacional, y de su análisis de la historia y de la naturaleza de las relaciones internacionales en tanto que construcción social. ${ }^{4}$ Las dos obras colectivas fueron el resultado de las reuniones del Comité Británico y en ellas se refleja el trabajo conjunto de la primera generación de la Escuela Inglesa clásica, ${ }^{5}$ mientras que The Anarchical Society planteó los principales temas en torno a los que se articularían los debates y trabajos de la segunda generación. ${ }^{6}$
*Elsa AIMÉ GONZÁLEZ, Profesora Asociada de la Universidad Pontificia de Comillas; investigadora del grupo de Estudios Africanos y del Grupo de Estudio de las Relaciones Internacionales de la Universidad Autónoma de Madrid.

\footnotetext{
BULL, Hedley, The Anarchical Society: a Study of Order in World Politics, Columbia University Press, Nueva York, 20I 2; SUGANAMI, Hidemi; CARR, Madeline y HUMPHREYS, Adam (eds.) The Anarchical Society at 40: contemporary challenges and prospects, Oxford University Press, Oxford, 2017.

2 GRASA HERNÁNDEZ, Rafael y GARCÍA SEGURA, Caterina (dirs.) Cambios en la Naturaleza de la Diplomacia y de la Guerra en los Cuarenta Años de la Sociedad Anárquica de Hedley Bull,Tirant Lo Blanch,Valencia, 2019.

3 Estas dos reediciones se publicarán por Oxford University Press; según se recoge en la página web de la editorial a fecha de I de junio de 2019, la fecha estimada de publicación de Diplomatic Investigations es septiembre de 2019, mientras que la fecha estimada para The Expansion of International Society es noviembre de 2020.

${ }^{4}$ BUZAN, Barry, An Introduction to the English School of International Relations, Polity, Cambridge, 20I4, p. I0.

5 Ibídem, pp. 6-7.

6 lbídem, p. 8.
}

DOI:

https://doi.org/I0.15366/relacionesinternacionales2019.4I.00I

Formato de citación recomendado:

AIMÉ GONZÁLEZ, Elsa, “La teoría de la sociedad internacional. De la narrativa clásica de Escuela Inglesa al enfoque crítico”, en Relaciones Internacionales, n 4I, 2019, pp. 13 - 31. 
La trayectoria y el enfoque de la teoría clásica de la sociedad internacional no permiten situarla de forma inequívoca entre las teorías clásicas de las Relaciones Internacionales. Surgió en el marco del debate entre realismo y liberalismo a finales de los años 1930 con el propósito de ofrecer una teoría a medio camino entre estas teorías. Aunque no logró del todo romper con ellas, ha estado más abierta que estas al diálogo con los enfoques reflectivistas, especialmente en el contexto de la post Guerra Fría, y de hecho su consolidación en el panorama teórico de las relaciones internacionales correrá parejo al del reflectivismo a partir de la década de 1980. Es cierto no obstante que tampoco es posible situarla en las teorías reflectivistas ya que, en sus inicios, no dialogó con estas ni llegó a formular una teoría crítica con el orden internacional existente en el momento en que surgió. Por ello, recibió algunas críticas que denigraron en bloque las aportaciones de lo que se bautizaría entonces como la Escuela Inglesa ${ }^{7}$, que negaron su existencia, ${ }^{8}$ o que por el contrario reclamaron una mayor apertura hacia otros enfoques $y$ teorías para desarrollar su potencial en lugar de estancarse en la defensa del orden establecido.9 Estas críticas reflejan la polarización entre los defensores de los enfoques de la teoría de la elección racional que permitió la convergencia del liberalismo y del realismo en sus versiones neo, y los enfoques reflectivistas hacia los cuales la Escuela Inglesa se mostraba más cercana por sus planteamientos ontológicos y epistemológicos. ${ }^{10}$

Es en este contexto que surgió lo que denominamos teoría crítica de la sociedad internacional. Una serie de autores con un enfoque reflectivista han iniciado un fructífero diálogo con los autores de la sociedad internacional conformando lo que denominamos aquí como la teoría crítica. Así se refleja por ejemplo en obras de reciente publicación como la de Suganami, Carr y Humphreys mencionada anteriormente, o como en The Globalization of International Society, editada por Tim Dunne y Christian Reus-Smit." En ese diálogo con el enfoque clásico, los autores críticos han retomado el concepto de sociedad internacional para repensar la trayectoria histórica atendiendo a sus disfunciones, su pluralidad, así como las jerarquías y desigualdades que la caracterizan.

El objetivo de este artículo es plantear algunos de los elementos característicos de estos dos enfoques, clásico y crítico, de la de la sociedad internacional.A tal fin, analiza, en primer lugar, el surgimiento de la Escuela Inglesa y de su primera generación; en segundo lugar, aborda los cambios de dicha escuela tras la Guerra Fría con su consolidación en el panorama teórico de las Relaciones Internacionales a través de una segunda generación de autores que prolongaron y actualizaron su agenda de investigación.; finalmente, examina la aparición de una teoría crítica de la sociedad internacional y los elementos característicos de la misma.

\footnotetext{
7 JONES, Roy E.,"The English School of international relations: a case for closure” en Review of International Studies, vol. 7, n I, I98I, pp. I-I3.

8 GRADER, Sheila, "The English school of international relations" en Review of International Studies, vol. I4, n I, 1989, pp. 29-44.

9 W/EVER, Ole, "International society - Theoretical promises unfulfilled?" en Cooperation and Conflict, vol. 27, n I, 1992, pp. 97- I 28.

${ }^{10}$ En el contexto del segundo debate Hedley Bull cuestionó los planteamientos positivistas que resurgirían en la síntesis neo-neo, cuya teoría de la elección racional no guarda relación con el racionalismo definido por Martin Wight característico de la Escuela Inglesa, y que defendió la importancia de los enfoques (auto)críticos. BULL, Hedley, “The case for a classical approach” en World Politics, vol. I8, $n^{\circ} 3$, 1966, pp. 36I-377.

" DUNNE,Tim y REUS-SMIT, Christian, (eds.) The Globalization of International Society, Oxford University Press, Oxford, 2017.
} 


\section{La Escuela Inglesa en sus inicios}

Aunque existen diferentes relatos acerca de los orígenes de la Escuela Inglesa ${ }^{12}$ todos coinciden en situar a algunos investigadores, especialmente a Martin Wight, Hedley Bull y Adam Watson en sus orígenes. También reconocen el papel decisivo que jugó la fundación en 1958 del British Committee on the Theory of International Politics por el historiador Herbert Butterfield. Sin embargo, la denominación 'Escuela Inglesa' genera confusión, no sólo porque no todos sus integrantes eran británicos ${ }^{13}$, sino también porque el Comité Británico dependió en sus inicios financieramente de la Fundación Rockefeller. Una primera advertencia es por tanto necesaria. Lo distintivo de esta corriente de pensamiento no es su pretendido carácter inglés, o británico, sino que se aplicase en desarrollar la idea de la existencia de una sociedad internacional de estados y, con ello, una comprensión de lo internacional que trascendía al estado. Rompía, asimismo, con la costumbre de pensar y enseñar las relaciones internacionales en términos de "asuntos exteriores" y de "problemas de política exterior". ${ }^{14}$ Así, estos autores se centraron en estudiar la política internacional, su funcionamiento desde una perspectiva teórica; en analizar la historia de las relaciones internacionales para sacar lecciones de cara a las necesidades del presente; $y$ en identificar el sustrato moral que entendían, cimentaba la sociedad internacional. ${ }^{15}$

Lo característico de los miembros del Comité Británico fue la creencia en que la disciplina iba más allá del debate entre idealismo y realismo ${ }^{16}$, tal y como se refleja en varios de los capítulos de la que podemos considerar como la primera obra clásica de esta corriente de pensamiento, Diplomatic Investigations. Essays in the Theory of International Politics editado por Herbert Butterfield y Martin Wight y publicado en 1966. Sin embargo, yendo más allá del ámbito académico, es importante mencionar que esta escuela surgió en un contexto de declive del imperio británico, cuestión que ayuda a comprender la agenda de investigación en sus inicios y su orientación teórica.

Paradójicamente el calificativo "inglesa” fue atribuido en un artículo crítico que planteaba una disociación entre las teorías que se hacían en Estados Unidos y en el Reino Unido, y que reclamaba el cierre de esta escuela —aunque tuvo el efecto contrario-17. Por ello, hablar de la teoría de la sociedad internacional para englobar los trabajos dispares que han analizado, desde un enfoque interpretativo y normativo, la naturaleza societaria de las relaciones internacionales y su transformación histórica es igualmente válido, con la ventaja de que esta denominación resalta su enfoque sociológico las relaciones internacionales. A fin de cuentas, más que participar en la

12 SUGANAMI, Hidemi, "The English school, history and theory” en Ritsumeikan International Affairs, vol. 9, n² 2, 20I I, pp. 29-30; NAVARRI, Cornelia, "Introduction: methods and methodology in the English school” en NAVARI, Cornelia, (ed.) Theorising International Society: English School Methods, Palgrave MacMillan, Hampshire, 2009 [I ${ }^{a}$ edición], p. 7.

13 Hedley Bull era australiano, mientras que Charles Manning, al que Hidemi Suganami y Cornelia Navari sitúan también en los orígenes de la Escuela inglesa era sudafricano.

14 WIGHT, Martin, "Why is there no international theory?" en BUTTERFIELD, Herbert y WIGHT, Martin, (eds.) Diplomatic Investigations. Essays in the Theory of International Politics, Harvard University Press, Cambridge, 1966, p. 21.

15 Véase BUTTERFIELD, Herbert y WIGHT, Martin, “Preface” en BUTTERFIELD, Herbert y WIGHT, Martin, (eds.) Diplomatic Investigations.

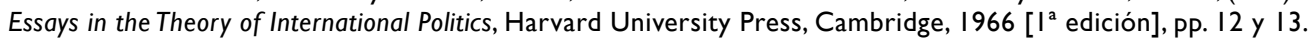

${ }^{16}$ LINKLATER, Andrew y SUGANAMI, Hidemi, The English School of International Relations. A Contemporary Reassessment, Cambridge University Press, Cambridge, 2006, pp. I56-I57.

17 JONES, Roy E., "The English school of international relations: a case for closure” op. cit. Lo cierto es que aunque el Comité Británico no eligió su nombre, sino que le vino impuesto por la Fundación Rockefeller, reivindicó pronto sus diferencias con respecto al Comité Americano, cf. BUTTERFIELD, Herbert y WIGHT, Martin, “Preface” en BUTTERFIELD, Herbert y WIGHT, Martin, (eds.) Diplomatic Investigations. op. cit., p. 12. Sobre la necesidad de reconocer, pero no esencializar, las diferencias entre las teorías de relaciones internacionales a partir de su ubicación física y teórica véase WÆVER, Ole, "The sociology of a not so international discipline: American and European developments in International Relations” en International Organization, vol. 52, n 4, 1998, pp. $687-727$. 
división geográfica entre academias, la teoría de la sociedad internacional vino a participar en los debates ontológicos, epistemológicos y metodológicos que estaban teniendo lugar en ciencias sociales, y en particular en la por entonces aún joven disciplina de Relaciones Internacionales.

Con un trabajo fuertemente marcado por la observación de la dimensión histórica de las relaciones internacionales, el enfoque de la Escuela Inglesa subrayó la importancia de nociones como paz, orden y justicia en las relaciones entre los estados, y la existencia de una estructura social compuesta por normas legales e instituciones. El objetivo era superar la imagen realista de las relaciones internacionales como la de un sistema caracterizado por una yuxtaposición de entidades políticas en colisión, predominante en aquella época y de raigambre estadounidense. Reexaminó esta lectura atendiendo a los vínculos surgidos de la convivencia entre los estados y a la sociedad que originaban más allá de las fronteras, sin asumir de partida la idea liberal de la armonía de intereses. Es decir, se puede decir que la Escuela Inglesa fue un efecto del primer debate de la teoría de Relaciones Internacionales en la medida en que uno de sus propósitos fue ofrecer una nueva teoría, a medio camino entre el realismo y el liberalismo.

Asimismo, frente al énfasis de las interpretaciones realistas o marxistas en la dimensión material de las relaciones internacionales, sea en términos de capacidad militar o económica de los estados occidentales, y a la interpretación de la expansión europea como fruto de la superioridad moral y material del liberalismo, la Escuela Inglesa llamó la atención sobre la importancia de analizar la estructura normativa internacional, su funcionamiento y su evolución histórica. Esta nueva perspectiva fue desarrollada en el contexto de la Guerra Fría por lo que denominamos aquí la primera generación de la teoría clásica de la sociedad internacional en la que, como ya hemos apuntado, incluimos entre otros autores a Hedley Bull,Adam Watson o Martín Wight.

La importancia que concedieron a la estructura normativa internacional refleja la impronta del derecho internacional en las obras de estos autores, como se ve también en su reformulación del concepto jurídico de "sociedad de naciones" como "sociedad internacional". ${ }^{18}$ De hecho,Wight defenderá este último concepto como una 'vía media' entre el liberalismo y el realismo al plantear que existe una moralidad política diferente de la moralidad individual — central para el primeroy de la (a)moralidad propia de la razón de estado — defendida por el segundo—. ${ }^{19}$ Para Wight, por el contrario, la conciencia social internacional y la moralidad internacional se retroalimentan en la medida en que esta última, a diferencia de la moralidad de las personas, no legitima el interés de los estados en toda circunstancia sino que posibilita la convivencia entre los estados, la búsqueda de la justicia internacional y el autocontrol estatal. Para este autor, como ocurre también con los demás miembros de la primera generación, la transformación de las relaciones internacionales desde la Paz de Westfalia (1648) hasta el presente se caracteriza, más bien, por una continuidad: la de la existencia de una sociedad internacional de estados con una moralidad propia. ${ }^{20}$

\footnotetext{
18 NIEMEYER, Gerhart, Law without Force. The Function of Politics in International Law, Transaction Publishers, New Brunswick y Londres, 200 I [ I ${ }^{\text {a }}$ edición 194I], pp. 289-293.

19 WIGHT, Martin, "Western values in International Relations” en BUTTERFIELD, Herbert y WIGHT, Martin, Diplomatic Investigations, op. cit., p. 91.

${ }^{20}$ WIGHT, Martin, “Western values in International Relations” en BUTTERFIELD, Herbert y WIGHT, Martin, Diplomatic Investigations, op.
} cit. 
Esta primera generación centró su reflexión histórica en la expansión europea decimonónica y en la evolución de la sociedad internacional desde entonces hasta las descolonizaciones. Esta corriente teórica enfatizó como rasgo distintivo de dicha sociedad internacional la existencia de normas y valores con unas raíces civilizatorias comunes. ${ }^{21}$ Así, según Wight: "la sociedad internacional existe en virtud de un núcleo de estándares y de costumbres comunes, difíciles de definir, pero encarnadas parcialmente en el derecho internacional". 22 Por su parte, para Hedley Bull, dicha sociedad se había fundamentado históricamente en la existencia de una cultura compartida, de un mismo sustrato intelectual y moral, que facilitaba la comunicación y reforzaba la percepción de unos intereses comunes. ${ }^{23}$

De ahí que la obra de Bull The Anarchical Society. A Study of Order in World Politics, publicada por primera vez en 1977, fuera un estudio del marco legal, las creencias morales y las instituciones de la sociedad internacional y de su impacto en el orden internacional. En ella ofreció una definición canónica:

"Una sociedad de estados (o sociedad internacional) sólo existe cuando un grupo de estados, conscientes de sus intereses y valores comunes, forman una sociedad en el sentido de que se consideran unidos por una serie de normas comunes, que regulan sus relaciones y de que colaboran en el funcionamiento de las instituciones comunes."24

Con esa definición, Bull concretó su concepto de sociedad internacional, en contraste con Wight, quien usaba los conceptos de sistema y sociedad indistintamente. Bull considera que, en un sistema internacional, los estados pueden "interactuar de tal forma que pasen a constituir factores que entrarán necesariamente en los cálculos de cada uno", mientras que la sociedad internacional sería una unión superior, más fuerte y compleja, cimentada en intereses, valores, normas e instituciones comunes, y posibilitada por la existencia de un sustrato cultural y civilizatorio compartido. ${ }^{25}$ Sin embargo, Bull también consideró que, tras las descolonizaciones, la sociedad internacional del siglo XX estaba en riesgo al carecer de cultura o civilización común. Sin embargo, dejó inexplorada esta aparente paradoja al centrar su obra en el análisis de una realidad particular: la de la sociedad europea de estados. ${ }^{26}$

Desde esta perspectiva, la percepción de la existencia de una cultura común propició que Occidente (entendiendo por ello la sociedad internacional compuesta por los estados europeos y aquellos en los que, por su pasado colonial, predominaba la cultural europea ${ }^{27}$ ) se diferenciara del mundo no occidental mediante un estándar de civilización. A través de dicho estándar Occidente estipuló los criterios (políticos, económicos, culturales) a los que se tenían

\footnotetext{
${ }^{21}$ O'HAGAN, Jacinta, Conceptualizing the West in International relations. From Spengler to Said, Palgrave, Nueva York, 2002 , p. II4.

${ }^{22}$ WIGHT, Martin, "Western values in International Relations” en BUTTERFIELD, Herbert y WIGHT, Martin, Diplomatic Investigations. Essays in the Theory of International Politics, Harvard University Press, Cambridge, 1966, p. 103.

${ }^{23}$ BULL, Hedley, La Sociedad Anárquica. Un Estudio sobre el Orden en la Política Mundial, Los Libros de la Catarata, Madrid, 2005 [I a edición en inglés 1977], p. 365

${ }^{24}$ BULL, Hedley, La Sociedad Anárquica... op. cit., p. 65. Énfasis añadido.

25 Ibídem, p. 65.

26 Ibídem, p. 67.

${ }^{27}$ O'HAGAN, Jacinta, Conceptualizing the West in International Relations... op. cit., p. I I 5.
} 
que ajustar las sociedades no occidentales que quisieran ver reconocida su plena soberanía y no ser sometidas a un trato desigual.

Según este relato, que denominamos teoría clásica de la sociedad internacional, la adaptación de esas sociedades no occidentales al estándar de civilización fue lo que transformaría, a partir de mediados del siglo $X X$, la sociedad internacional occidental en una sociedad internacional global que, sin embargo, mantuvo los criterios occidentales para la conducta de las relaciones internacionales. Por ello, este enfoque entiende la sociedad internacional global como una creación occidental. ${ }^{28}$ Esta idea fue desarrollada en la obra colectiva The Expansion of International Society, publicada por primera vez en 1984 y editada por Bull y Watson. Más adelante, en el marco de la post Guerra Fría, surgió como veremos a continuación una segunda generación que centró su reflexión en la evolución de la sociedad internacional tras las descolonizaciones y en la reaparición del estándar de civilización tras la década de 1990. ${ }^{29}$ Esa segunda generación retomó la reflexión que Bull dejó pendiente de desarrollar acerca de la sociedad internacional del siglo $X X$ y su transformación.

\section{La evolución de la Escuela Inglesa tras la Guerra Fría}

En la década de 1990 surgió una nueva generación que, retomando los planteamientos de la primera, actualizó su enfoque al calor de las transformaciones ligadas al fin de la Guerra Fría. ${ }^{30}$ Aunque, como ha señalado Hidemi Suganami, la década de 1990 fue relativamente tranquila para la teoría de la sociedad internacional si tenemos en cuenta su producción en las décadas anteriores, ${ }^{31}$ también fue un periodo clave para su consolidación. Hasta entonces, las obras de la Escuela Inglesa clásica se habían centrado fundamentalmente en el mundo hasta las descolonizaciones, ${ }^{32}$ mientras que las relaciones internacionales de la década de 1990 se entendían como caracterizadas por la globalización. Terminada la Guerra Fría, surgió una reflexión acerca de los nuevos desafíos a los que se enfrentaba la sociedad internacional, entre otros aspectos, en relación con el mantenimiento de orden o la promoción de la justicia. En esta discusión subyacía un interrogante sobre sí el marco normativo internacional creado para regular las relaciones entre los estados europeos y producto de unas sociedades con unos valores culturales compartidos - surgido desde este punto de vista en el seno de la civilización occidental - permitía gestionar la diversidad cultural característica de la sociedad internacional contemporánea en la que convivían diferentes civilizaciones.

${ }^{28}$ Ibídem, p. 125.

${ }^{29}$ La distinción de varias generaciones dentro de la Escuela Inglesa es frecuente si bien, aunque la distinción de una primera generación parece más o menos clara (con discrepancias sobre algunos de los primeros fundadores, como veremos), no hay un consenso en la distinción de generaciones posteriores, ni se han analizado suficientemente sus características específicas. Buzan y Suganami coinciden por ejemplo en distinguir dos generaciones correspondientes a antes y después del fin de la Guerra Fría, aunque no llegan a aclarar los rasgos característicos de la segunda; Yongjin Zhang señala sin embargo que la tarea de la segunda generación se ha articulado en torno a cuatro ejes: reconstruir, representar y reposicionar la Escuela Inglesa, y crear redes; Cornelia Navari distingue por su parte hasta tres generaciones de la teoría de la sociedad internacional antes del fin de la Guerra Fría; la distinción de una teoría crítica de la que hablamos a continuación tampoco ha generado consenso. BUZAN, Barry, An Introduction to the English School of International Relations, op. cit., p. 8; SUGANAMI, Hidemi, "The historical development of the English School” en NAVARI, Cornelia y GREEN, Daniel, (eds.) Guide to the English School in International Studies, Wiley Blackwell, West Sussex, 20I4, p. I6. ZHANG, Yongjin, "The global diffusion of the English School" en NAVARI, Cornelia y GREEN, Daniel, (eds.) Guide to the English School... op. cit., Pp. 232234. NAVARI, Cornelia "English School methodology” en NAVARI, Cornelia y GREEN, Daniel, (eds.) Guide to the English School... op. cit., p. 2II. Distinguimos por tanto dos generaciones de la teoría clásica de la sociedad internacional, con algunos autores a caballo entre ambas (como Donnelly, Gong o Suganami).

30 BUZAN, Barry, “The English School: an underexploited resource in IR" en Review of International Studies, vol. $27, \mathrm{n}^{\circ}$ 3, $200 \mathrm{I}, \mathrm{p} .473$.

3I SUGANAMI, Hidemi, "The historical development of the English School” en NAVARI, Cornelia y GREEN, Daniel M., (eds.) Guide to the English School... op. cit., p. 15.

32 BUZAN, Barry, From International to World Society? English School Theory and the Social Structure of Globalisation, Cambridge University Press, Cambridge, 2004, pp. 17, 22 
La segunda generación de la teoría clásica de la sociedad internacional se volcó en profundizar en las ideas y enfoque de sus predecesores de la Escuela Inglesa sobre la sociedad internacional y el orden mundial. ${ }^{33}$ Prolongaron la reflexión al mundo posterior a 1945 y especialmente al mundo globalizado de la post Guerra Fría. Como señala Caterina García Segura, ${ }^{34}$ ello supuso un proceso de renovación de sus conceptos centrales y un acercamiento al constructivismo social que había irrumpido con gran fuerza en la disciplina desde mediados de los ochenta con el que compartían un fuerte interés por la dimensión identitaria y cultural de las relaciones internacionales.

\section{I. La consolidación de la teoría de la sociedad internacional en la post Guerra Fría} El número especial que publicó en 1992 la revista Millennium refleja la consolidación y renovación de la agenda de investigación de la Escuela Inglesa, con varios artículos que proponían un mayor intercambio con otras teorías, y que buscaban actualizarla atendiendo a los cambios en el contexto internacional. ${ }^{35}$ Tres factores ayudan a comprender por qué esta escuela que no había tenido cabida en el debate interparadigmático al cuestionarse precisamente que fuese una teoría ${ }^{36}$ cobró tanta relevancia con el fin de la Guerra Fría.

En primer lugar, el hecho de que a lo largo de la década de 1980 la Escuela Inglesa hubiese comenzado una reflexión sobre sí misma, su historia e identidad, ${ }^{37}$ permitió consolidar su marco interpretativo desde el que analizar el fin de la Guerra Fría y sus consecuencias. El libro de Timothy Dunne Inventing International Society: A History of the English School es representativo en este sentido tanto de la reflexión de dicha escuela sobre sí misma en tanto que corriente teórica, como del creciente interés en la disciplina de Relaciones Internacionales por sus aportaciones y diálogo con otras corrientes teóricas. ${ }^{38}$

En segundo lugar, en la post Guerra Fría resurgió un interés académico (en especial desde enfoques reflectivistas) por la cuestión cultural en las relaciones internacionales, y en particular por la manera en que la civilización en tanto que idea conforma las identidades de los actores internacionales y condiciona el orden y las estructuras normativas de la sociedad internacional (tanto en el pasado como en el contexto de la post Guerra Fría). ${ }^{39}$ Los trabajos de la Escuela

${ }^{33}$ LINKLATER, Andrew y SUGANAMI, Hidemi, The English School of International Relations. A Contemporary Reassessment, Cambridge University Press, Cambridge, 2006, p. 25

${ }^{34}$ GARCÍA SEGURA, Caterina, “La Escuela Inglesa y la teoría de la sociedad internacional: propuestas, críticas y reformulación” en DEL ARENAL, Celestino y SANAHUJA, José Antonio, Teorías de las Relaciones Internacionales, Tecnos, Madrid, 2015, p. 286.

${ }^{35}$ Cf. Millennium - Journal of International Studies, vol. 21, n 3, 1992. Los artículos de Chris Brown y de Tony Evans y Peter Wilson reflejan esos intercambios entre la Escuela Inglesa y otras teorías, como el liberalismo y la teoría de los regímenes; los de Nicholas J. Rengger M. J. Peterson, Ronnie D. Lipschutz y Martin Shaw muestran el interés por repensar los postulados de la Escuela Inglesa clásica en el contexto de la globalización; y los artículos de Fred Halliday, Nicholas J. Wheeler y Steve Smith reflejan el interés por profundizar en sus planteamientos teóricos y conceptuales.

${ }^{36}$ Dos artículos clásicos de las críticas que se plantearon a la Escuela Inglesa en la década de 1980 son el de Roy Jones que, como se suele señalar, paradójicamente bautizó la Escuela Inglesa con ese nombre, y el de Sheila Grader que cuestionó su existencia.Véanse: JONES, Roy E., "The English school of International Relations..." op. cit.; GRADER, Sheila, "The English school of International Relations: evidence and evaluation" en Review of International Studies, vol. I4, n I, 1988, pp. 29-44.

${ }^{37}$ Lo que Hidemi Suganami denomina "el debate sobre la Escuela Inglesa", en LINKLATER, Andrew y SUGANAMI, Hidemi, The English School..., op. cit., pp. 17-25.

38 DUNNE, Timothy, Inventing International Society. A History of the English School, MacMillan, Londres, I998. Desde la publicación de la obra de Dunne, varios libros han ahondado en la reflexión sobre la epistemología y ontología de la Escuela Inglesa, entre otros: NAVARI, Cornelia, (ed.) Theorising International Society... op. cit.; NAVARI, Cornelia y GREEN, Daniel, (eds.) Guide to the English School.. op. cit.; BUZAN, Barry, An Introduction to the English School... op. cit.;

39 Una de las obras que contribuyeron a este resurgir fue el artículo y posterior libro de Samuel Huntington sobre El Choque de Civilizaciones, si bien otras obras han adoptado una mirada crítica sobre esta cuestión, especialmente desde principios del siglo 
Inglesa publicados en los años setenta, ochenta y noventa del siglo $X X$ sobre la expansión de la sociedad europea de estados y el surgimiento de nuevos estados y actores internacionales, así como sobre su transformación como consecuencia de "la revuelta contra Occidente", ${ }^{40}$ ya habían llamado la atención sobre esa dimensión identitaria de las relaciones internacionales y ofrecían un corpus desde el que repensarla y con el que dialogar desde otras teorías. ${ }^{41}$

En tercer lugar, y ligado a lo anterior, durante la Guerra Fría la confrontación entre la Unión Soviética y Estados Unidos acaparó la atención de las escuelas dominantes entonces en la disciplina -en especial del (neo)realismo- en detrimento de otros procesos que se estaban dando como la propia descolonización y su impacto en las relaciones internacionales. En la medida en que la Escuela Inglesa sí había prestado atención a este proceso, ${ }^{42}$ sus trabajos cobraron relevancia tanto para comprender el nuevo orden internacional de la post Guerra Fría, como para profundizar en la comprensión de la evolución histórica de la sociedad internacional tras las independencias. En la post Guerra Fría, la atención de la segunda generación de la escuela se centró en la existencia y características de los nuevos estados surgidos en el periodo posterior a la Segunda Guerra Mundial y su evolución tras el fin de la Guerra Fría.Algunos investigadores y analistas políticos señalaron su debilidad o fragilidad estructural, su dependencia económica, sus reiteradas vulneraciones de los derechos humanos y el (sub)desarrollo de sus poblaciones. Pasaban a plantear que esos estados, ahora denominados frágiles, fallidos o colapsados, representaban una nueva amenaza para el orden y la estabilidad internacional. ${ }^{43}$

Estos tres factores contribuyeron al afianzamiento de la Escuela Inglesa con el fin de la Guerra Fría. Aunque a principios de la década de 1990 pudiese parecer que dicha escuela estaba cuestionada y en declive, su agenda de investigación fue cobrando protagonismo. ${ }^{44}$ La apertura de nuevos debates en Relaciones Internacionales y un creciente diálogo con las corrientes reflectivistas, especialmente el constructivismo social, contribuyeron a revigorizarla y consolidarla como una perspectiva pertinente para comprender la estructura y el funcionamiento de la sociedad internacional en perspectiva histórica. Ello redundará en el afianzamiento de su agenda de investigación en torno a seis grandes áreas de trabajo: primero, la historia de la sociedad internacional $-y$ especialmente el encuentro de determinados países no occidentales con la "familia de naciones civilizadas"-; segundo, las instituciones de la guerra y el equilibrio de poder dentro de la sociedad internacional; tercero, los debates en torno al orden y la justicia,y, por tanto, sobre el papel de la ética y la moralidad de la sociedad internacional,y su relación con el derecho

XXI, como señala SCHULZ, Carsten-Andreas, "Civilisation, barbarism and the making of Latin America's place in 19th-century international society” en Millennium. Journal of International Relations, vol. 42, $\mathrm{n}^{\circ}$ 3, p. 845. Jackson también plantea una reflexión cosmopolita sobre la civilización en la post Guerra Fría, en JACKSON, Robert H., Quasi-states: Sovereignty, International Relations and the Third World, Cambridge University Press, Cambridge, I990., pp. I42-I 43.

${ }^{40}$ BULL, Hedley, "The revolt against the West" en BULL, Hedley y WATSON, Adam, (eds.) The Expansion of International Society, Clarendon Press, Oxford, 1985 [I ${ }^{\text {a }}$ edición 1984], pp. 21 7-228.

${ }^{41}$ EPP, Roger, "The English school on the frontiers of international society: a hermeneutic recollection" en Review of International Studies, vol. $24, n^{\circ} 5,1998$, p. 49.

42 Ibídem.

${ }^{43}$ El artículo paradigmático de este tipo de pensamiento fue "The coming anarchy" de Robert D. Kaplan, que afirmaba la inviabilidad de los nuevos estados e incluso la necesidad de restaurar la figura del fideicomiso. Cf. KAPLAN, Robert D., "The coming anarchy" en The Athlantic Monthly, febrero de 1994, pp. 44-76.

${ }^{44}$ REUS-SMIT, Christian, "The constructivist challenge after September II" en BELLAMY, Alex J., International Society and its Critics, Oxford University Press, Oxford, 2005, pp. 8I-82. 
internacional, así como con cuestiones concretas como intervenciones militares, derechos humanos y minorías; cuarto, la reflexión sobre la propia escuela, sus conceptos y trayectoria; quinto, la relación con otras teorías de Relaciones Internacionales y con la teoría Política; y finalmente, la reflexiones en torno al estado, la soberanía y la diplomacia, así como el papel de la ideología y las revoluciones. ${ }^{45}$

\subsection{La agenda de investigación en la post Guerra Fría}

La renovación conceptual y teórica de la segunda generación de la Escuela Inglesa supuso profundizar en cuestiones que ya habían sido advertidas en The Anarchical Society y en The Expansion of International Society, y en concreto en el funcionamiento, cohesión y perspectivas de futuro de la sociedad internacional desde el fin de la dominación europea. ${ }^{46}$ Se interpretó la transformación de la estructura internacional tras 1945 como el debilitamiento de la sociedad internacional de estados, fruto de la aparición de estados débiles y de la división ideológica propia de la contienda bipolar. El posterior colapso de la Unión Soviética —entendido como la victoria del liberalismose interpretó, a su vez, como la posibilidad de alcanzar un nuevo orden global que contrarrestase los problemas derivados del creciente pluralismo y diversidad cultural existente en el seno la sociedad internacional, gracias a "un movimiento hacia una sociedad internacional más solidarista", retomando los términos de la Escuela Inglesa. ${ }^{47}$

En un mundo que ya no se encontraba polarizado en torno a dos bloques, resurgió la reflexión planteada unos años antes por Hedley Bull (primero en su texto "The Grotian conception of international society" 48 y luego en la tercera parte de The Anarchical Society) acerca de los posibles futuros de la sociedad internacional con dos posibles escenarios. Un posible escenario sería una evolución hacia una sociedad mundial, cohesionada en torno a un conjunto de valores y propósitos compartidos a escala planetaria, definiendo unas normas comunes que permitieran mantener el orden y promover la justicia internacional —enfoque solidarista-. Otro posible escenario era una sociedad internacional compuesta por una multiplicidad de estados que coexisten gracias a un consenso de mínimos sobre las reglas que rigen sus relaciones y que les permiten perseguir diferentes objetivos —enfoque pluralista-.

En la obra de Bull, los dos enfoques comparten la idea de una sociedad internacional compuesta por estados, legalmente articulada, con obligaciones compartidas, y que vela por su preservación. Difieren sin embargo en hasta dónde se debe o puede regular algunas instituciones fundamentales como la guerra, en cuál es la fuente del derecho internacional, y en el estatus en la sociedad internacional de los individuos frente al de los estados. ${ }^{49}$ Así, mientras que el pluralismo considera que, para preservar el orden internacional, ha de prevalecer la protección

\footnotetext{
${ }^{45}$ BUZAN, Barry, "The English School: an underexploited resource in IR" en Review of International Studies, vol. 27, $\mathrm{n}^{\circ} 3$, 200 I, pp. 47I488.

${ }^{46}$ BULL, Hedley, La Sociedad Anárquica... op. cit., ps364-366; BULL, Hedley y WATSON, Adam, (eds.) The Expansion of International Society, op. cit., pp. 430-435; WATSON, Adam, The Evolution of International Society, Routledge, Londres y Nueva York, 1992, pp. 307-309.

${ }^{47}$ HURRELL, Andrew, "Order and justice” en NAVARI, Cornelia y GREEN, David, (eds.) Guide to the English School in International Studies, Wiley Blackwell, West Sussex, 20I4, p. 149.

${ }^{48}$ BULL, Hedley, “The Grotian conception of international society” en BUTTERFIELD, Herbert yWIGHT, Martin, Diplomatic Investigations... op. cit., pp. 5 I-73.

${ }^{49}$ BAIN, William, “The pluralist-solidarist debate in the English School” en NAVARI, Cornelia y GREEN, Daniel, (eds.) Guide to the English School... op. cit., p. 159; LINKLATER, Andrew y SUGANAMI, Hidemi, The English School of International relations... op. cit., p. 60.
} 
de la soberanía de los estados, el solidarismo prioriza la protección de los seres humanos. ${ }^{50}$

Este debate, ligado a la forma y posibilidad de la acción moral en las relaciones internacionales, resurgió con el fin de la Guerra Fría y las diferentes interpretaciones de la misma. ${ }^{51}$ Desde una perspectiva optimista y liberal, se interpretó como la victoria de Occidente, y la posibilidad de superar la fragmentación cultural y la confrontación en torno a valores éticos características del periodo anterior. ${ }^{52}$ El nuevo orden mundial propiciaba avanzar hacia el solidarismo al alcanzarse un mayor consenso en torno a los valores liberales y a la necesidad de actuar en coalición para defenderlos (como mostraban tanto la Guerra del Golfo (1990-91) como las intervenciones humanitarias de principios de la década de 1990. Por el contrario, desde un prisma pesimista inclinado hacia el realismo, ponía el énfasis en la aparición de nuevos conflictos armados, la reemergencia del nacionalismo, y las consecuencias negativas de la globalización (económicas, demográficas, ecológicas, entre otros aspectos). El nuevo orden mundial evidenciaba por ello la dificultad para avanzar hacia una mayor integración de los estados, por lo que otros autores de la segunda generación defendían la necesidad de que, en la sociedad internacional, se protegiera el pluralismo, manteniendo las reglas de coexistencia que habían permitido durante siglos la convivencia entre los estados. ${ }^{53}$

El resurgimiento del debate pluralismo-solidarismo dentro de la segunda generación de la escuela significó un retorno de la reflexión acerca de si era posible y deseable llegar a un acuerdo sobre los estándares morales de la sociedad internacional, ${ }^{54} \mathrm{y}$, en consecuencia, sobre las características de la sociedad internacional contemporánea y su posible evolución. El debate se centró en gran medida en torno a los derechos humanos, las intervenciones humanitarias y la responsabilidad de Occidente con respecto al denominado Tercer Mundo, ${ }^{55}$ cuestiones ligadas a su vez al funcionamiento del régimen internacional de soberanía. Según se interpretó, el enfrentamiento bipolar había sido propicio para la supervivencia de algunos estados a pesar de sus debilidades estructurales gracias al apoyo que recibieron de las superpotencias a cambio de su alineamiento ideológico. Con el fin de la Guerra Fría esas alianzas desaparecieron originando un "proceso de descomposición de muchos estados" que se caracterizaría tanto por la aparición de nuevos estados independientes (como consecuencia de la desmembración de la URSS, o en África con la independencia de Eritrea) así como como por la proliferación de conflictos internos y graves crisis de los derechos humanos (en especial en los países surgidos de la descolonización). ${ }^{56}$ Todo ello contribuiría a "erosionar la mitología del estatalidad" como un absoluto moral y generaría una "crisis de legitimidad de los estados postcoloniales". ${ }^{57}$

\footnotetext{
${ }^{50}$ LINKLATER, Andrew y SUGANAMI, Hidemi, The English School of International relations... op. cit., pp. 64-65.

${ }^{51}$ REUS-SMIT, Christian, "The constructivist challenge after September II" en BELLAMY, Alex J., International Society and its Critics, op. cit., p. 86; HALLIDAY, Fred, Las Relaciones Internacionales en un Mundo en Transformación, Los Libros de la Catarata, Madrid, 2002, PP. 26I-262; RUIZ-GIMÉNEZ ARRIETA, Itziar, La Historia de la Intervención Humanitaria. El Imperialismo Altruista, Los Libros de la Catarata, Madrid, 2005, p. II 3.

${ }^{52}$ SØRENSEN, Georg, "IR theory after the Cold War" en Review of International Studies, vol. 24, n 5, 1998, p. 100.

${ }^{53}$ Véase por ejemplo JACKSON, Robert H., The Global Covenant: Human Conduct in a World of States, Oxford University Press, Oxford, 2000.

${ }^{54}$ WATSON, Adam, The Evolution of International Society, Routledge, Londres y Nueva York, I992, p. 307; BELLAMY, Alex J., International Society and its Critics, op. cit., p. 10.

${ }^{55}$ BUZAN, Barry, From International to World Society?... op. cit., p. 46

${ }^{56}$ RUIZ-GIMÉNEZ ARRIETA, Itziar, La Historia de la Intervención Humanitaria... op. cit., pp. I2I - I 22.

57 Ibídem, pp. 123-124, 137.
} 
El análisis de Robert H. Jackson Quasi-States: Sovereignty, International Relations and the Third World ${ }^{58}$ fue pionero de esta discusión característica de la post Guerra Fría. Aunque es una obra de 1990, la enmarcamos dentro de los trabajos de la segunda generación de la Escuela Inglesa clásica en tanto que parte de la narrativa de la primera generación sobre la expansión europea para analizar sus consecuencias con la instauración de lo que entiende como un nuevo régimen internacional de soberanía tras la descolonización. Según Jackson, ese nuevo régimen supuso la sustitución del estándar de civilización decimonónico por un estándar de soberanía excesivamente laxo. Se suplantaba la idea de soberanía positiva por la de soberanía negativa, al permitir la supervivencia de nuevos estados estructuralmente débiles y con gobiernos conflictivos o irresponsables, a pesar de que generaban una amenaza a la cohesión internacional. En su opinión, el estándar de civilización habría servido hasta entonces para determinar qué países merecían el reconocimiento como estados soberanos, pero la revuelta contra Occidente produjo un cambio con el que se abandonó la conexión entre el reconocimiento de la soberanía y la estatalidad empírica en favor de una estatalidad jurídica, instaurada desde arriba, es decir, por los estados independientes ya existentes. ${ }^{59}$

Desde este punto de vista, la descolonización supuso la creación de una estructura jurídica internacional que amparó la existencia de estados débiles sin estructuras propias consolidadas, denominados por ello “cuasi-estados". Retomando la diferenciación de Isaiah Berlin entre libertad positiva y negativa, Jackson calificó ese nuevo régimen internacional de soberanía como negativo - a diferencia del anterior que entendía como positivo-, al considerar que garantizaba a los estados la no interferencia en sus asuntos internos, permitiendo su independencia al margen de sus capacidades; no se basaba por tanto en los atributos políticos de los estados sino en un entramado legal e institucional no-competitivo que garantizaba el derecho a existir a todos los estados. ${ }^{60}$

El paso de un régimen de soberanía positivo a uno negativo constituye para Jackson una etapa más de la historia de la sociedad de estados soberanos que comienza con la Paz de Westfalia, símbolo del fin de la unidad de la república cristiana medieval bajo una misma autoridad papal frente a la aparición de una diversidad de gobiernos soberanos seculares e independientes. ${ }^{61}$ Desde este punto de vista,Westfalia supuso la aparición en Europa de la soberanía como institución política, ${ }^{62}$ sobre la que se ha construido una asociación de estados que excluyó a los sistemas políticos no europeos y permitió que se ejerciera sobre ellos una soberanía imperial.

Daniel Philpott abundaría posteriormente en esta lectura al interpretar la Paz de Westfalia como una revolución que originó el propio régimen internacional de soberanía, al señalar a los

\footnotetext{
${ }^{58}$ JACKSON, Robert H., Quasi-States: Sovereignty, International Relations and the Third World, Cambridge University Press, Cambridge, 1990.

59 JACKSON, Robert H., Quasi-States... op. cit., p. 5.

60 lbídem, p. 24.

${ }^{61}$ JACKSON, Robert H., "Sovereignty in world politics: a glance at a conceptual landscape" en JACKSON, Robert H., (ed.) Sovereignty at the Millennium, Blackwell, Oxford, 1999, pp. 16-19.

62 Ibídem, p. 20. Jackson detalla que dicha soberanía podía adoptar diferentes formas: dinástica (cuando residiese en reyes o familias reales), imperial (cuando fuese ejercida desde un poder imperial y mediante agentes coloniales), parlamentaria (cuando residiese en parlamentos o asambleas) o popular (cuando fuese ejercida por el conjunto de la ciudadanía de un país).
} 
estados soberanos como la autoridad política legítima. ${ }^{63}$ En sintonía con los planteamientos de la Escuela Inglesa clásica, Philpott planteó que, con Westfalia, se estableció una distinción entre las entidades políticas soberanas y las que no lo eran -que casualmente coincidían con las entidades políticas no europeas, aunque Philpott no se interrogaría sobre ello-. Una distinción fundamentada originalmente sobre el criterio de su cristiandad, pero que evolucionaría hasta formularse como un estándar de civilización con el que, en el siglo XIX, se distinguió entre los estados europeos/occidentales que conformaban la sociedad internacional y el resto del mundo que quedaba fuera ${ }^{64}$ Así, desde esta perspectiva la aparición de la soberanía marca una ruptura con el periodo medieval en la articulación política de Europa, pero en la relación entre Europa y el mundo no europeo pervivió la lógica medieval de la superioridad de la cristiandad latina, transformada posteriormente en el imaginario de la civilización occidental: "La vieja frontera medieval entre la cristiandad y el mundo no cristiano fue redefinida como una línea entre el mundo occidental civilizado y el resto del mundo, aún no suficiente o correctamente civilizado.”65

Jackson entiende por tanto que, entre la Paz de Westfalia y el fin de la Segunda Guerra Mundial, la sociedad internacional se basó en un régimen de soberanía positivo, en el que la independencia política estaba vinculada a la capacidad de gobierno real. Este periodo terminaría con tras la Segunda Guerra Mundial furto de la revuelta contra Occidente que provocaría la desaparición del estándar de civilización, dando paso a un nuevo periodo en el que la soberanía se habría desvinculado de los atributos de gobierno. ${ }^{66}$ Para Philpott este cambio supuso una segunda revolución en el régimen internacional de soberanía al transformar el marco normativo internacional e instituir la igualdad jurídica de los pueblos; ${ }^{67}$ rompió el régimen de soberanía positivo anterior, en el que el estándar de civilización servía de vara para medir quién tenía derecho a pertenecer a la sociedad internacional y quién no, y por tanto para decidir qué sociedad podía ser colonizada y cuál no. Si hasta entonces el estándar de civilización había jugado un papel clave en el régimen de soberanía positivo al vincular la civilización a la independencia soberana, ${ }^{68}$ con la descolonización se deslegitimaron las jerarquías inherentes a la sagrada misión civilizadora. La condición de sujeto colonizado pasaría así, según este enfoque, a sustituir a la civilización como requisito para el reconocimiento de nuevos estados soberanos, consolidando el principio del uti possidetis iuris como la base para reclamar la autodeterminación. ${ }^{69}$

La transformación del régimen de soberanía con las descolonizaciones supuso para Jackson una revolución en la moralidad europea al abandonar su paternalismo. ${ }^{70}$ Sin embargo, también considera que trajo la aparición de un desfase entre la igualdad de iure y las profundas desigualdades de facto - materiales y morales - entre los estados. Es lo que Jackson denomina el "dilema de los cuasi estados", un dilema normativo surgido de la coexistencia, en la práctica

\footnotetext{
${ }^{63}$ PHILPOTT, Daniel, Revolutions in Sovereignty. How Ideas Shaped Modern International Relations, Princeton University Press, Princeton y Oxford, 200I, pp. 3-4.

${ }^{64}$ Ibídem, pp. 32 y ss.

${ }^{65}$ JACKSON, Robert H., Quasi-States... op. cit., p. 2 I; PHILPOTT, Daniel, Revolutions in Sovereignty... op. cit., p. 35.

${ }^{66}$ JACKSON, Robert H., Quasi-States... op. cit., capítulo 4.

${ }^{67}$ PHILPOTT, Daniel, Revolutions in Sovereignty... op. cit., p. 4.

68 Ibídem, pp. 74-78.

69 JACKSON, Robert H., "Sovereignty in world politics..." op. cit., pp. 24-27.

70 JACKSON, Robert H., Quasi-States... op. cit., p. 85.
} 
del régimen de soberanía positivo con el negativo. ${ }^{71}$ Desde este punto de vista, la independencia de los nuevos estados se fundamentó en el derecho a la autodeterminación y de no intervención, pero también se acompañó de la creación de un régimen internacional con el que promover su desarrollo y cimentar su estatalidad, y por lo tanto con el que promover su soberanía positiva. Se habría instaurado así un régimen de soberanía específico para los estados surgidos de la descolonización, sustentados a través de la ayuda internacional al desarrollo y protegidos por las normas internacionales que impedirían una intervención directa en sus asuntos internos. Sin embargo, en el contexto de la post Guerra Fría, la democracia y los derechos humanos cobraron protagonismo como indicadores de buen gobierno y como nueva fuente de legitimidad de la autoridad política. Se impulsaron así nuevos proyectos internacionales de construcción de la soberanía positiva, tanto con operaciones de construcción de la paz como mediante la condicionalidad política de la ayuda. ${ }^{72}$

Desde esta óptica, el régimen de soberanía erigido en el Tercer Mundo con el colapso del colonialismo europeo se entendía como una solución temporal y artificial al problema de la incorporación de nuevas áreas del globo al sistema internacional erigido tras Westfalia, que presentaba disfuncionalidades que habían de ser resueltas para garantizar el orden y la gobernanza global. Así, en el marco de la revitalización del debate político y académico sobre la soberanía, algunas voces dentro de la segunda generación de la Escuela Inglesa defendieron que esta no podía ser ya un principio absoluto. ${ }^{73}$ Es en este contexto que surgió a comienzos del siglo XXI el principio de la responsabilidad de proteger que, junto a las intervenciones humanitarias, dará lugar a diversas reflexiones teóricas dentro de la teoría de la sociedad internacional. ${ }^{74}$

\section{La crítica a la teoría clásica de la sociedad internacional}

En el contexto de la post Guerra Fría, y de la mano de los diferentes giros que se produjeron en la teoría de las Relaciones Internacionales -histórico, sociológico, normativo, interpretativo, etc. - surgieron diferentes críticas desde las corrientes reflectivistas al relato desarrollado por las dos primeras generaciones de la teoría de la sociedad internacional. ${ }^{75}$ Diversos autores se comenzaron a problematizar las desigualdades y jerarquías internacionales existentes en la sociedad internacional.También desarrollaron una reflexión crítica sobre el estándar de civilización, su función y fundamentos. Desde este punto de vista, el relato clásico de la Escuela Inglesa se caracteriza por su sesgo eurocéntrico ${ }^{76}$ tanto en su interpretación de la expansión europea y del surgimiento de la sociedad internacional global, como en los planteamientos normativos derivados de esta lectura.

\footnotetext{
71 Ibídem, pp. I35-I37.

72 RUIZ-GIMÉNEZ ARRIETA, Itziar, La Historia de la Intervención Humanitaria... op. cit., p. I 37.

${ }^{73}$ Sobre la naturaleza de la soberanía en el Tercer Mundo y sus disfunciones, véase CLAPHAM, Christopher, "Sovereignty and the Third World state" en JACKSON, Robert H., (ed.) Sovereignty at the Millenium, Blackwell Publishers, Oxford, I999 [Iª edición], pp. I00-I I5. Clapham fue una de esas voces que planteó que la soberanía no podía mantenerse como el principio organizativo por antonomasia de la sociedad internacional globalizada.

74 Véase entre otros WHEELER, Nicholas J., Saving Strangers. Humanitarian Intervention in International Society, Oxford University Press, Oxford, 2000;WELSH, Jennifer M., Humanitarian Intervention and International Relations, Oxford University Press, Oxford, 2004.

75 BELLAMY, Alex J., International Society and its Critics, op. cit., pp. 7-8.

${ }^{76}$ HOBSON, John M., The Eurocentric Conception of World Politics. Western International Theory, I 760-20 I0, Cambridge University Press, Cambridge, 20I2, pp. 222-233.
} 
Esta mirada crítica señaló cómo al situar a Occidente en el centro del relato la Escuela Inglesa ha contribuido a invisibilizar la agencia de las sociedades no occidentales, especialmente su capacidad para cuestionar, modificar y resignificar las normas e instituciones internacionales. Frente a ello varios autores visibilizarán los silencios del relato clásico sobre la expansión europea, especialmente con respecto a la violencia de la expansión. Igualmente abordaran el análisis del papel que los aspectos ideacionales como la religión, la raza y el género han jugado en dicha expansión. Esta mirada alternativa que aspira, en suma, a superar el eurocentrismo de la teoría clásica, es la que denominamos en esta tesis como teoría crítica de la sociedad internacional. ${ }^{77}$

Algunos de los rasgos de la teoría clásica de la sociedad internacional abrieron la puerta a la posterior aparición de la teoría crítica de la sociedad internacional. Podemos destacar, en primer lugar, su esfuerzo por superar el pensamiento dicotómico de las teorías clásicas, cuestionando la lógica dialéctica del primer debate entre el liberalismo y el realismo —presente también en el marxismo - en favor de un planteamiento más dialógico. ${ }^{78}$ En segundo lugar, el posicionamiento metodológico de la Escuela Inglesa es sumamente crítico con el behaviorismo y ha reivindicado la importancia de la historia y de la filosofía como bases para la (auto)crítica y para el enjuiciamiento de los problemas teóricos de las Relaciones Internacionales. ${ }^{79}$ Martin Wight por ejemplo rechazó el conductismo afirmando que el estudio de las relaciones internacionales "no es un proceso de análisis científico; es más parecido a la crítica literaria." ${ }^{80}$ En tercer lugar, al cuestionar la forma de pensar de las teorías clásicas y su pensamiento construido sobre opuestos como soberanía y anarquía, al Escuela Inglesa reclamaba una mayor atención sobre las relaciones entre ambos conceptos, como se refleja en el propio concepto de sociedad anárquica con el que Bull buscó desenclavar el pensamiento tal y como reclamaría posteriormente el postestructuralismo. En cuarto lugar, al llamar la atención sobre las normas e instituciones que ordenan la sociedad internacional, su surgimiento y transformación, la primera generación antecede a las teorías que han reivindicado de forma explícita el tiempo histórico para pensar las relaciones internacionales como es el caso de la sociología histórica internacional, el postestructuralismo o el constructivismo. En quinto lugar, la teoría clásica de la sociedad internacional converge con las teorías que han reclamado un mejor entendimiento de cómo se conforman y cambian las estructuras y agentes internacionales no sólo en términos materiales sino también ideacionales como ocurre por

\footnotetext{
77 Acerca de la distinción entre una literatura clásica sobre la expansión europea en la Escuela Inglesa, y el desarrollo de una mirada crítica que amplía y revisa ese relato, véase BUZAN, Barry, An Introduction to the English School of International Relations, Polity, Cambridge, 2014, pp. 60-77. En este trabajo subrayamos como característico de este enfoque crítico, que se ha comenzado a desarrollar en los últimos años, su alejamiento de los postulados eurocéntricos de la teoría clásica. HOBSON, John M., The Eurocentric Conception of World Politics..., p. 223.

78 Mientras que el pensamiento dialéctico se articula sobre una triada (tesis, antítesis, síntesis) entendida como un lucha entre posiciones contrarias que se resuelve mediante la trascendencia, el pensamiento dialógico se articula sobre el reconocimiento de los contrarios como elementos complementarios y reconoce la contradicción en lugar de cuestionarla, por lo que no busca resolverla sino entenderla en tanto que proceso e intercambio que permite la transformación; véase por ejemplo el planteamiento de Julia Kristeva recogido en NEUMANN, Iver B., "Self and other in International Relations" en European Journal of International Relations, vol. 2, $n^{\circ}$ 2, 1996, pp. 149-150. Varios autores han apuntado la idea de que la Escuela Inglesa se plantea en términos dialógicos, especialmente Roger Epp al señalar los vínculos del pensamiento de Wight con la hermenéutica dialógica de Gadamer (y la influencia en este de Collingwood). Epp remite a su vez a Richard Shapcott, y este a su vez a Andrew Linklater; sin embargo, en la práctica la dimensión dialógica de la Escuela Inglesa ha sido poco estudiada hasta la fecha. Cf. EPP, Roger, "The English school on the frontiers of international society: a hermeneutic recollection" en Review of International Studies, vol. 24, n 5, 1998, pp. 47-64.

79 BULL, Hedley, “The case for a classical approach" en World Politics, vol. I8, n 3, 1966, ps. 36I-377. El artículo está disponible en castellano: BULL, Hedley, "Teoría internacional: El caso de

un enfoque clásico" en Relaciones Internacionales, $\mathrm{n}^{\circ}$ 38, 2018, pp. 191-204, disponible en: https://revistas.uam.es/index.php/ relacionesinternacionales/article/view/9765/0

${ }^{80}$ WIGHT, Martin, International Theory. The Three Traditions, Holmes and Meier, Nueva York, I 992, p. 258.
} 
ejemplo en el constructivismo, la teoría crítica, el feminismo, o el postcolonialismo. Estas teorías reflectivistas coinciden con la teoría crítica de la sociedad internacional en analizar las normas e instituciones internacionales en perspectiva histórica para apreciar la capacidad de agencia de los estados (también de los estados desempoderados) y de otros actores no-estatales en la creación y transformación de su entorno.

Sin embargo, a pesar de tener elementos en común con varias teorías reflectivistas, la teoría clásica de la sociedad internacional también ha recibido importantes críticas por parte de estas, especialmente en relación con los silencios y distorsiones generados por su interpretación de la historia de las relaciones internacionales $y$, en particular, del surgimiento, expansión y transformación de la sociedad internacional.Así, han planteado la necesidad de revisar la expansión como un proceso de homogeneización originado en Europa y basado en los valores liberales, para tener mucho más en cuenta la interacción con los actores no europeos. La teoría crítica aboga por el contrario por analizar la agencia y capacidad de estos últimos para transformar la sociedad internacional, sus normas e instituciones. También han reclamado una mirada más crítica sobre los mecanismos de poder de carácter no material, y sobre las disfunciones y contradicciones de la sociedad internacional ya que estas son clave en la articulación de los cambios en y de las resistencias a relaciones de dominación que, aunque silenciadas por la Escuela inglesa, caracterizan la expansión europea.

Esta lectura crítica ha sido desarrollada, por ejemplo, desde un enfoque postcolonial, por Siba N. Grovogui quien señala que el análisis de Jackson es incompleto porque minusvalora los mecanismos de gobernanza global que han limitado las capacidades de los estados surgidos con la descolonización, y porque ignora las desigualdades estructurales del sistema internacional actual. Así, centrándose en la realidad de los estados africanos, señala la responsabilidad occidental en la configuración de las estructuras de poder africanas. ${ }^{81}$ Aunque Grovogui reconoce la pertinencia de la preocupación de Jackson por el fracaso del estado, considera que su razonamiento asume que los problemas políticos y la mala gestión económica en los denominados cuasi-estados se deben a que los estados africanos se desvían de las tradiciones occidentales de constitucionalismo, tolerancia política y pluralismo democrático. De esta forma, según señala Grovogui,Jackson legitima que sean los estados occidentales los que determinen los estándares de conducta a los que otros han de someterse, y los que intervengan en defensa de esos valores. Por tanto, según señala Grovogui, en vez de prestar atención a cuáles han sido las consecuencias del orden internacional en los estados africanos, la lectura de la Escuela Inglesa recalca los problemas de estos como estrictamente internos, generando una paradoja según la cual los gobernantes africanos ejercen un control soberano pero, al mismo tiempo, se cuestiona que realmente lo ejerzan. ${ }^{82}$

La crítica de Grovogui al relato de Jackson nos sirve para poner de manifiesto la pervivencia dentro de la segunda generación de la teoría clásica de la sociedad internacional de la narrativa eurocéntrica presente en la primera. Así, las interpretaciones de Jackson y Philpott, entre otros, y que apuntamos anteriormente, no sólo se construyen sobre una interpretación de la historia internacional como la de un núcleo original (la sociedad europea westfaliana) que se expande,

\footnotetext{
${ }^{81}$ GROVOGUI, Siba N., "Regimes of sovereignty: international morality and the African condition" en European Journal of International Relations, vol. 8, $\mathrm{n}^{\circ}$ 3, 2002, pp. $318-322$.

82 Ibídem.
} 
como ocurría en la primera generación, sino que su propuesta normativa busca contribuir a que ese núcleo originario se mantenga como centro normativo de la sociedad internacional. ${ }^{83}$

Tal y como ha señalado Itziar Ruiz-Giménez, esta narrativa es ilustrativa de la reemergencia tras la Guerra Fría de una sociedad internacional articulada en círculos concéntricos, con un centro occidental asociado a los principios de orden y justicia, y una periferia que encarna la inestabilidad y las violaciones de los derechos humanos. Así, a partir de la década de 1990 parece resurgir:

"la antigua dualidad de la sociedad internacional en la que no todos los Estados son reconocidos como soberanos, ni todos disfrutan del derecho de no-intervención. Algunos pueden incluso verse sometidos a intervención o supervisión internacional hasta que sus gobernantes, o pueblos, cumpliesen los estándares internacionales de civilización." ${ }^{4}$

Según plantea Ruiz-Giménez en La Historia de la Intervención Humanitaria. El Imperialismo Altruista, la emergencia durante la Guerra Fría de un régimen internacional de los derechos humanos $^{85}$, y la interpretación del fin de la Guerra Fría como la victoria de Occidente y del orden liberal propiciarán la reactivación de la doctrina de la guerra justa que había legitimado las intervenciones hasta las descolonizaciones, formulada no ya en términos de civilización sino de defensa de los derechos humanos, de la democracia y del sistema de seguridad colectiva de la ONU. ${ }^{86}$

Ruiz-Giménez señala sin embargo que es posible identificar en esta transformación la prolongación de las viejas lógicas civilizatorias, una continuidad particularmente visible en las políticas de cooperación al desarrollo y de asistencia humanitaria con las que se tratará de impulsar la paz democrática y de abandonar el régimen de soberanía negativo en favor de una soberanía responsable. ${ }^{87}$ Para esta autora, el cuestionamiento en la post Guerra Fría de la soberanía de los estados que no se guían por principios democráticos y de respeto de los derechos humanos, dará a un nuevo régimen de intervención articulado en torno a la proliferación de intervenciones militares humanitarias y de operaciones de mantenimiento de la paz como las de Somalia o Ruanda entre otras. Todo ello evidenciaría el resurgimiento del estándar civilizatorio y de la dualidad de la sociedad internacional que habían caracterizado la sociedad internacional hasta el fin del colonialismo. ${ }^{88}$

En este sentido la teoría crítica de la sociedad internacional presta especial atención a la

\footnotetext{
${ }^{83}$ Tal vez como consecuencia de las críticas vertidas al enfoque eurocéntrico de Quasi-states, una década después Jackson replanteó su interpretación de la descolonización y de la desaparición del estándar de civilización frente al principio de autodeterminación como la solución para permitir el reconocimiento de sistemas políticos no occidentales. Cf. JACKSON, Robert H., The Global Covenant, op. cit., p. 409. En todo caso, la crítica de Grovogui pone asimismo de manifiesto el nexo poder/conocimiento en tanto que evidencia cómo algunos trabajos normativos de la segunda generación de la Escuela Inglesa clásica han alimentado el propio discurso que se estaba dando en relaciones internacionales para legitimar las intervenciones humanitarias.

${ }^{84}$ RUIZ-GIMÉNEZ ARRIETA, Itziar, La Historia de la Intervención Humanitaria... op. cit., p. 142.

${ }^{85}$ Ibídem, pp. 76-79.

86 Ibídem, pp. 48, II5.

87 Ibídem, pp.II7-II 8 y 139-141.

${ }^{88}$ Ibídem, pp. |4|-| 43.
} 
prolongación y transformación de las estructuras de dominación de las relaciones internacionales. Si la teoría clásica de la sociedad internacional ha jugado un papel central en la conjugación de la teoría de las Relaciones Internacionales con la historia internacional, a lo largo de los últimos veinte años diversas críticas han convergido en señalar que la Escuela Inglesa ha reproducido el pensamiento dual de la sociedad europea de estados. Desde un enfoque crítico, la teoría clásica distorsiona la comprensión de la sociedad internacional porque la concibe más como un proceso de difusión unidireccional del centro a la periferia que como una interacción social.

Así, según plantea Jacinta O’Hagan, aunque en la Escuela Inglesa es visible una concepción multicivilizacional de las relaciones internacionales, sus autores conciben Occidente como el principal actor en la articulación moderna de la relación entre las diferentes civilizaciones a través de las estructuras de la sociedad internacional, ${ }^{89} \sin$ comprender cómo estas se conmstituyen social y políticamente. Para John M. Hobson contrarrestar la lógica dual de la teoría clásica de la sociedad internacional requiere partir de interpretaciones no eurocéntricas del sistema internacional y de la economía política global. ${ }^{90}$ Edward Keene por su parte señala que es necesario prestar más atención a los patrones asociativos y a las relaciones en la sociedad internacional en lugar de focalizar toda la atención en la estructura y en la idea de la "entrada" de nuevos actores en la sociedad internacional. ${ }^{91}$ Una idea compartida por Carsten A. Schulz quien afirma que la revisión de esta narrativa permite también trascender la idea de Occidente frente al resto.9 ${ }^{92}$ En otras palabras, la revisión crítica de la teoría de la sociedad internacional cuestiona que los imaginarios sociales de los actores de las relaciones internacionales reflejen identidades necesariamente inflexibles y hostiles unas con otras, y subraya que la construcción delYo y del Otro es un proceso fundamentalmente ideológico, al servicio de determinados proyectos políticos que cristalizan en el derecho internacional. ${ }^{93}$

\section{Conclusión}

En la post Guerra Fría, y particularmente desde comienzos del siglo XXI, se ha consolidado la mirada histórica y sociológica de lo internacional reivindicada por Martin Wight hace cincuenta años como necesaria para ver la existencia de una sociedad internacional. ${ }^{94}$ Medio siglo después, ese enfoque sociohistórico se ha ampliado al calor de las investigaciones de la teoría de la sociedad internacional y de las teorías reflectivistas. Mientras que algunos autores como Buzan o Jackson han buscado profundizar desde dentro de la Escuela Inglesa en los planteamientos normativos y conceptuales, otros como Grovogui o Ruiz-Giménez han entablado desde el reflectivismo un diálogo que ha originado una teoría crítica de la sociedad internacional.

Esta mirada crítica al relato clásico de la sociedad internacional ha planteado la necesidad

\footnotetext{
${ }^{99}$ O'HAGAN, Jacinta, Conceptualizing the West in International Relations... op. cit., p. I08. Según O'Hagan este enfoque muestra la impronta en la Escuela Inglesa de la obra de Arnold Toynbee.

${ }^{90}$ HOBSON, John M., "The twin self-delusions of IR: why 'hierarchy' and not 'anarchy' is the core concept of IR" en Millennium. Journal of International Studies, vol. 42, $\mathrm{n}^{\circ}$ 3, 20I4, ps. 557-575.

9 KEENE, Edward, "The standard of 'civilisation', the expansion thesis and the 19th century international social space" en Millennium. Journal of International Relations, vol. 42, $\mathrm{n}^{\circ} 3,2014$, pp. 667-672.

92 SCHULZ, Andreas-Carsten, “Civilisation, barbarism and the making of Latin America...” op. cit., p. 844.

93 LEBOW, Richard Ned, “Identity and international relations” en International Relations, vol. 22, n 4, 2008 , p. 488.

94 WIGHT, Martin, "Western values in International Relations” en BUTTERFIELD, Herbert y WIGHT, Martin, Diplomatic Investigations, Harvard University Press, Cambridge, 1966, pp. 89-130.
} 
de profundizar en el fértil terreno planteado por el enfoque clásico, pero prestando atención a la desigualdad, las jerarquías y la coerción inherentes al orden liberal. ${ }^{95} \mathrm{En}$ los últimos años es posible ver cómo esta discusión se ha afianzado, tanto a través de la reedición de las obras clave de la teoría clásica como por la aparición de nuevas reflexiones que han tratado de refinar el enfoque clásico, o de revisarlo desde un enfoque crítico. La teoría de la sociedad internacional ha sido en este sentido mucho más receptiva a las críticas constructivas que le han sido planteadas desde el reflectivismo que a las que sencillamente han asumido su irrelevancia, algo que se refleja en la orientación de sus trabajos desde el fin de la Guerra Fría.

\section{Bibliografía}

BELLAMY, Alex J., BELLAMY,Alex J., International Society and its Critics, Oxford University Press, Oxford, 2005.

BULL, Hedley, “The case for a classical approach” en World Politics, vol. 18, n 3, I966, ps. 36I-377.

BULL, Hedley, La Sociedad Anárquica. Un Estudio sobre el Orden en la Política Mundial, Los Libros de la Catarata, Madrid, 2005 [I a edición en inglés 1977].

BULL, Hedley, The Anarchical Society: a Study of Order in World Politics, Columbia University Press, Nueva York, 2012.

BULL, Hedley y WATSON, Adam, (eds.) The Expansion of International Society, Clarendon Press, Oxford, 1985 [I edición 1984].

BUTTERFIELD, Herbert y WIGHT, Martin, (eds.) Diplomatic Investigations. Essays in the Theory of International Politics, Harvard University Press, Cambridge, 1966 [I ${ }^{\text {a }}$ edición].

BUZAN, Barry, "The English School: an underexploited resource in IR" en Review of International Studies, vol. 27, $\mathrm{n}^{\circ} 3$, 200I, pp. 47I-488.

BUZAN, Barry, An Introduction to the English School of International Relations, Polity, Cambridge, 2014.

BUZAN, Barry, From International to World Society? English School Theory and the Social Structure of Globalisation, Cambridge University Press, Cambridge, 2004.

CLAPHAM, Christopher, "Sovereignty and the Third World state" en JACKSON, Robert H., (ed.) Sovereignty at the Millenium, Blackwell Publishers, Oxford, 1999, pp. I00-II5.

DUNNE, Timothy, Inventing International Society. A History of the English School, MacMillan, Londres, 1998.

DUNNE, Tim y REUS-SMIT, Christian, (eds.) The Globalization of International Society, Oxford University Press, Oxford, 2017

EPP, Roger, "The English school on the frontiers of international society: a hermeneutic recollection" en Review of International Studies, vol. 24, n 5, 1998, pp. 47-64.

GARCÍA SEGURA, Caterina, "La Escuela Inglesa y la teoría de la sociedad internacional: propuestas, críticas y reformulación” en DELARENAL, Celestino y SANAHUJA, JoséAntonio, Teorías de las Relaciones Internacionales, Tecnos, Madrid, 20I5, pp. 269-299.

GRADER, Sheila, “The English school of International Relations: evidence and evaluation” en Review of International Studies, vol. I4, n I, 1988, pp. 29-44.

GRASA HERNÁNDEZ, Rafael y GARCÍA SEGURA, Caterina (dirs.) Cambios en la Naturaleza de la Diplomacia y de la Guerra en los Cuarenta Años de la Sociedad Anárquica de Hedley Bull,Tirant Lo Blanch,Valencia, 2019.

GROVOGUI, Siba N., "Regimes of sovereignty: international morality and the African condition" en European Journal of International Relations, vol. 8, $\mathrm{n}^{\circ}$ 3, 2002, pp. 3I5-338.

HALLIDAY, Fred, Las Relaciones Internacionales en un Mundo en Transformación, Los Libros de la Catarata, Madrid, 2002.

HOBSON, John M., "The twin self-delusions of IR: why 'hierarchy' and not 'anarchy' is the core concept of IR" en Millennium. Journal of International Studies, vol. 42, n 3, 20I 4, ps. 557-575.

HOBSON, John M., The Eurocentric Conception of World Politics. Western International Theory, I 760-20 I0, Cambridge University Press, Cambridge, 2012.

JACKSON, Robert H., "Sovereignty in world politics: a glance at a conceptual landscape" en JACKSON, Robert H., (ed.) Sovereignty at the Millennium, Blackwell, Oxford, 1999, pp. 9-34.

JACKSON, Robert H., Quasi-States: Sovereignty, International Relations and the Third World, Cambridge University Press, Cambridge, 1990.

JACKSON, Robert H., The Global Covenant: Human Conduct in a World of States, Oxford University Press, Oxford, 2000. JONES, Roy E., "The English School of international relations: a case for closure" en Review of International Studies, vol. 7, n I, 198I, pp. I-13.

KAPLAN, Robert D.,"The coming anarchy” en The Athlantic Monthly, febrero de 1994, pp. 44-76.

KEENE, Edward, "The standard of 'civilisation', the expansion thesis and the I9th century international social space"

\footnotetext{
95 HURREL, Andrew, “Order and justice” en NAVARI, Cornelia y GREEN, Daniel, (eds.) Guide to the English School... op. cit., pp. I5I, I52.
} 
en Millennium. Journal of International Relations, vol. 42, $\mathrm{n}^{\circ}$ 3, 20I4, ps. 65I-673.

LEBOW, Richard Ned, “Identity and international relations” en International Relations, vol. 22, n 4, 2008, pp. 473-492.

LINKLATER, Andrew y SUGANAMI, Hidemi, The English School of International Relations. A Contemporary Reassessment, Cambridge University Press, Cambridge, 2006.

NAVARI, Cornelia, (ed.) Theorising International Society: English School Methods, Palgrave MacMillan, Hampshire, 2009.

NAVARI, Cornelia y GREEN, Daniel, (eds.) Guide to the English School in International Studies, Wiley Blackwell, West Sussex, 2014.

NEUMANN, Iver B., "Self and other in International Relations" en European Journal of International Relations, vol. 2, $\mathrm{n}^{\circ}$ 2, 1996, pp. I39-174.

NIEMEYER, Gerhart, Law without Force. The Function of Politics in International Law, Transaction Publishers, New Brunswick y Londres, 200I [I Ia edición 194I], pp. 289-293.

O'HAGAN, Jacinta, Conceptualizing the West in International relations. From Spengler to Said, Palgrave, Nueva York, 2002.

PHILPOTT, Daniel, Revolutions in Sovereignty. How Ideas Shaped Modern International Relations, Princeton University Press, Princeton y Oxford, 200I.

RUIZ-GIMÉNEZ ARRIETA, Itziar, La Historia de la Intervención Humanitaria. El Imperialismo Altruista, Los Libros de la Catarata, Madrid, 2005

SCHULZ, Carsten-Andreas, "Civilisation, barbarism and the making of Latin America's place in 19th-century international society” en Millennium. Journal of International Relations, vol. 42, n 3, pp. 837-859.

SØRENSEN, Georg, "IR theory after the Cold War" en Review of International Studies, vol. 24, n 5, I998, pp. 83- 100.

SUGANAMI, Hidemi, “The English school, history and theory” en Ritsumeikan International Affairs, vol. 9, n’ 2, 20I I, pP. 27-50.

SUGANAMI, Hidemi; CARR, Madeline y HUMPHREYS,Adam (eds.) The Anarchical Society at 40: contemporary challenges and prospects, Oxford University Press, Oxford, 2017.

WÆVVER, Ole, "International society - Theoretical promises unfulfilled?" en Cooperation and Conflict, vol. 27, $\mathrm{n}^{\circ} \mathrm{I}$, 1992, pp. 97-I 28.

WÆVER, Ole,"The sociology of a not so international discipline:American and European developments in International Relations” en International Organization, vol. 52, n 4, 1998, pp. 687-727.

WATSON, Adam, The Evolution of International Society, Routledge, Londres y Nueva York, 1992.

WELSH, Jennifer M., Humanitarian Intervention and International Relations, Oxford University Press, Oxford, 2004.

WHEELER, Nicholas J., Saving Strangers. Humanitarian Intervention in International Society, Oxford University Press, Oxford, 2000.

WIGHT, Martin, International Theory. The Three Traditions, Holmes and Meier, Nueva York, 1992. 


\section{RELACIONES INTERNACIONALES}

Revista académica cuatrimestral de publicación electrónica Grupo de Estudios de Relaciones Internacionales (GERI)

Universidad Autónoma de Madrid, España

https://revistas.uam.es/relacionesinternacionales

ISSN 1699 - 3950

f facebook.com/RelacionesInternacionales

3. twitter.com/RRInternacional 